\title{
A Privacy Protection User Authentication and Key Agreement Scheme Tailored for the Internet of Things Environment: PriAuth
}

\author{
Yuwen Chen, José-Fernán Martínez, Pedro Castillejo, and Lourdes López \\ Departamento de Ingeniería Telemática y Electrónica (DTE), Escuela Técnica Superior de Ingeniería y Sistemas de Telecomunicación \\ (ETSIST), Universidad Politécnica de Madrid (UPM), C/Nikola Tesla, s/n, 28031 Madrid, Spain
}

Correspondence should be addressed to Yuwen Chen; yuwen.chen@upm.es

Received 6 July 2017; Revised 29 October 2017; Accepted 7 November 2017; Published 24 December 2017

Academic Editor: Anton Kos

Copyright (C) 2017 Yuwen Chen et al. This is an open access article distributed under the Creative Commons Attribution License, which permits unrestricted use, distribution, and reproduction in any medium, provided the original work is properly cited.

\begin{abstract}
In a wearable sensor-based deployment, sensors are placed over the patient to monitor their body health parameters. Continuous physiological information monitored by wearable sensors helps doctors have a better diagnostic and a suitable treatment. When doctors want to access the patient's sensor data remotely via network, the patient will authenticate the identity of the doctor first, and then they will negotiate a key for further communication. Many lightweight schemes have been proposed to enable a mutual authentication and key establishment between the two parties with the help of a gateway node, but most of these schemes cannot enable identity confidentiality. Besides, the shared key is also known by the gateway, which means the patient's sensor data could be leaked to the gateway. In PriAuth, identities are encrypted to guarantee confidentiality. Additionally, Elliptic Curve Diffie-Hellman $(\mathrm{ECDH})$ key exchange protocol has been adopted to ensure the secrecy of the key, avoiding the gateway access to it. Besides, only hash and XOR computations are adopted because of the computability and power constraints of the wearable sensors. The proposed scheme has been validated by BAN logic and AVISPA, and the results show the scheme has been proven as secure.
\end{abstract}

\section{Introduction}

As sensors become widespread in their usage regarding health monitoring scenarios, a significant amount of personal sensitive data like blood pressure, pulse, or electrocardiogram readings will be monitored. These sensors could be interconnected to compose a Wireless Body Area Network (WBAN). With different sensors gathering patient's data and continually sending these data to doctors or to a remote monitoring station for further analysis, it is necessary to make sure that these data are transferred confidentially. The usual way is to encrypt them first before they are sent. The proposal presented in this paper, named PriAuth, aims to help the patient and the doctor build a shared key for encrypting health parameters.

Because only appointed doctors are allowed to access the patient's data, the patient and the doctor have to authenticate each other first. A workable way is to introduce a gateway to help the patient authenticating the legitimacy of the doctor and vice versa. After authentication, the two parties will build a shared key for further communication.

When a doctor wants to read patient's data, he sends a request to the patient. The patient forwards this request together with his own identification information to the gateway. The gateway checks whether the patient and the doctor are legitimate, and if any of them is not regarded as such then the scheme is aborted. Only when they are all legitimate, the gateway sends the authentication result to the patient. Once the patient has become aware of the legitimacy of the doctor, he sends the authentication result to the doctor as well. Based on the authentication result, the patient and the doctor can build a shared key, which is used for encrypting confidential information sent between them.

There are many research results focusing on the authentication and key agreement problems; while most of them could ensure the safety of the data, this is not enough, as there is also a need to protect privacy. 
In the authentication process, the patient and the doctor have to send their identities and some other related information to the gateway. It has to be ensured that the patient's identity should not be leaked. Of course, a patient is usually unwilling to leak his identity information, because if the patient's identity is leaked, the health history and status of the patient will be freely available for anyone in the system, regardless of the patient wishes.

On the other hand, when a doctor sends his identity to the gateway for authentication, we have to make sure that the doctor's identity is kept confidential, too (e.g., when an adversary eavesdrops the identity of the doctor and finds out the doctor's major is dermatology according to the identity of the doctor, there is a great chance that the patient has a skin related problem). Therefore, it is also necessary to keep the doctor's identity confidential in order to protect the privacy of the patient. In PriAuth, Elliptic Curve Cryptography (ECC) is adopted as the method used to protect the identities of the data transmission participants, which is similar to [15-21].

After the gateway finishes the authentication process, the gateway will send the authentication result to the patient and the doctor. Based on the authentication result, the patient and the doctor could build a shared key. In some traditional schemes, the gateway could learn the key shared from the authentication information it gets from the patient and the doctor. This means the patient's personal health data could be leaked to the gateway. It is necessary to prevent the gateway learning this key. In PriAuth, Elliptic Curve Diffie-Hellman $(\mathrm{ECDH})$ key exchange protocol is adopted to ensure the shared key secrecy between the patient and doctor. Besides, only hash and XOR operations are adopted, which is suitable for the wearable sensors.

PriAuth has been validated by BAN logic and AVISPA. BAN logic is one of the most prevalent methods that help determine whether the exchanged information is trustworthy, secure against eavesdropping. BAN logic is also adopted to prove the security of the schemes by [22-24]. AVISPA (Automated Validation of Internet Security Protocols and Applications) is a tool for the automated validation of Internet security-sensitive protocols and applications, which has been widely adopted by [24-26], and so forth.

This paper is organized as follows: Section 2 is related works; Section 3 is the preliminary knowledge. In Section 4, we introduce PriAuth; Section 5 provides the BAN logic validation. Section 6 includes AVISPA verification. Section 7 is the security analysis part. Section 8 provides a comparison with other schemes. Section 9 is the validation part. Section 10 concludes with a summary of the contributions.

\section{Related Works}

In several papers of the researched literature, the authors use different acronyms; user and sensor are the most commonly used, which equals to doctor and sensor in our scheme. Thus, from now on, we will use user and sensor instead of doctor and patient. D. Wang and P. Wang provide overviews of some of the schemes described in $[27,28]$. Farash et al. use a single shared key between all the users or sensors to encrypt the identities [13]. All the sensors use the same key $h\left(X_{\mathrm{GWN}} \| 1\right)$ to encrypt the sensor identity, using XOR method where SID $_{j}$ is the sensor identity and $T_{2}$ is a timestamp.

$$
\mathrm{ESID}_{j}=\operatorname{SID}_{j} \oplus h\left(h\left(X_{\mathrm{GWN}} \| 1\right) \| T_{2}\right),
$$

where $h\left(X_{\mathrm{GWN}} \| 1\right)$ is a key that is shared by all the sensors, so malicious or curious sensors could learn the identity of sensor $\operatorname{SID}_{j}$. As $\operatorname{ESID}_{j}, T_{2}$ are sent via a public channel. A malicious or curious sensor with identity $\mathrm{SID}_{k} \mathrm{can}$ eavesdrop sensor $\operatorname{SID}_{j}$ to get $\operatorname{ESID}_{j}, T_{2}$. In order to get the sensor id $\mathrm{SID}_{j}, \mathrm{SID}_{k}$ could decrypt ESID $j$ using the same key $h\left(X_{\mathrm{GWN}} \| 1\right)$ :

$$
\begin{aligned}
\mathrm{ESID}_{j} \oplus h\left(h\left(X_{\mathrm{GWN}} \| 1\right) \| T_{2}\right) \\
=\left\{\operatorname{SID}_{j} \oplus h\left(h\left(X_{\mathrm{GWN}} \| 1\right) \| T_{2}\right)\right\} \\
\quad \oplus h\left(h\left(X_{\mathrm{GWN}} \| 1\right) \| T_{2}\right)=\mathrm{SID}_{j} .
\end{aligned}
$$

$\mathrm{Lu}$ et al. use a random identity $\mathrm{TID}_{i}$ to protect identity privacy [10]. But as the identity is a fixed value, a user could be tracked by an adversary. Schemes [29-32] use a similar method, but all these procedures are prone to suffer from tractability attack.

In scheme proposed by $\mathrm{Wu}$ et al., every time the gateway gives a new $\mathrm{PID}_{\text {newMU }}$ for the user [4]. But in this case, there is a potential loss of synchronization problem: if the adversary blocks the $\mathrm{PID}_{\text {newMU }}$ from being sent to the user, then the two parties may lose their synchronization. Das et al. protect the identity of the user by generating a new masked identity every time in a similar way, but this scheme suffers from loss of synchronization problem, too [33].

Jung et al. use the similar method with the scheme [13] of Farash et al. [6]. The key to encrypt the identity of a single user is the same for all the users. This scheme has the same problem that has been discussed. What a user sends to the gateway node is as follows: $\mathrm{DID}_{i}=h\left(\mathrm{ID}_{i} \| R_{1}\right), k=h\left(\mathrm{DID}_{i} \|\right.$ $\left.v^{*} \| T_{1}\right), A_{i}=E_{k}\left(\mathrm{DID}_{i}\left\|R_{1}\right\| T_{1}\right)$, so other users could learn DID $_{i}$ by decrypting $A_{i}$ with the same key $v^{*}$. Besides, this scheme has the same inner side attacker problem, a detailed analysis is shown in Section 7.4.

Rabin cryptosystem with quadratic residue problem is used to encrypt a message $[11,34]$. Assume $n=p q$, where $p$ and $q$ are two large primes. If $y=x^{2} \bmod n$ has a solution, that is, there exists a square root for $y$, then $y$ is called a quadratic residue $\bmod n$. The set of all quadratic residue numbers in $[1, n-1]$ is denoted by $\mathrm{QR}_{n}$. The quadratic residue problem states that, for $y \in \mathrm{QR}_{n}$, it is hard to find $x$ without the knowledge of $p$ and $q$ due to the difficulty of factoring $n$ [35]; this is a kind of public-key encryption method.

Chatterjee and Das provide a similar methodology of protecting the identity of the user. They use the ECC based public key methods [15]. Besides, they try to combine the authentication scheme with an attributed based access control scheme. He et al. use a similar method, while they use exponentiation operations instead [36].

We summarize some of them in Table 1 . From the table, it can be inferred that privacy is a problem that has not drawn enough attention from the researchers. In some schemes, 
TABLE 1: Comparison of protection of privacy.

\begin{tabular}{lccc}
\hline Schemes & Sensor anonymity & User anonymity & Shared key privacy \\
\hline Choi et al. [1] & $\times$ & $\times$ & $\sqrt{ }$ \\
Shi and Gong [2] & $\times$ & $\times$ & $\sqrt{ }$ \\
Chang and Le [3, Scheme 1] & $\times$ & $\times$ & $\times$ \\
Chang and Le [3, Scheme 2] & $\times$ & $\times$ & $\sqrt{ }$ \\
Wu et al. [4] & $\sqrt{ }$ & $\times$ \\
Das et al. [5] & $\sqrt{ }$ & $\times$ \\
Jung et al. [6] & $\sqrt{ }$ & $\times$ \\
Fan et al. [7] & $\times$ & $\times$ & $\times$ \\
Amin and Biswas [8] & $\times$ & $\times$ & $\sqrt{ }$ \\
Nam et al. [9] & $\times$ & $\times$ & $\times$ \\
Lu et al. [10] & $\sqrt{ }$ & $\times$ \\
Zhao et al. [11] & $\sqrt{ }$ & $\times$ \\
Hou et al. [12] & $\times$ & $\times$ & $\times$ \\
Farash et al. [13] & $\times$ & $\times$ & $\times$ \\
Turkanović et al. [14] & $\times$ & $\times$ & $\times$ \\
PriAuth & $\sqrt{ }$ & $\times$ & $\sqrt{ }$ \\
\hline
\end{tabular}

all the users share the same key to encrypt their identities, this means the encrypted identity could be decrypted by a malicious or curious user using the same key $[5,6,10,13]$. Some of the schemes fail to enable the anonymity of the user or sensor, such as [37-39]. We adopt the ECC based method to enable the anonymity, which is similar to [1521] because "ECC requires smaller keys compared to nonECC cryptography (based on plain Galois fields) to provide equivalent security" [40]. The gateway has a public key that is known by every user; all the identities are encrypted by an XOR method with a new key which is generated from gateway's public key before the identities are sent to the gateway. Thus, only the gateway could learn the identities.

As for the shared key between user and sensor, in some schemes, the gateway knows the shared key in schemes $[6-8,11-14]$, while, in some others, the gateway does not know the key, they use Diffie-Hellman (DH) anonymous key agreement protocol to build the shared key $[1,2,4,5,9,30]$. As we have discussed, the gateway is not allowed to know the shared key in order to prevent a curious gateway from eavesdropping the sensor data.

\section{Preliminary}

Elliptic Curve Cryptography (ECC) is a public-key cryptography approach based on the algebraic structure of elliptic curves over finite fields. For current cryptographic purposes, an elliptic curve is a plane curve over a finite field (rather than the real numbers) which consists of the points satisfying the following:

$$
y^{2}=x^{3}+a x+b
$$

In order to use ECC, all parties must agree on all the domain parameters of the elliptic curve $\{p, a, b, G, n, h\}$ :

$F(p)$ : the finite field over $p$, where $p$ is a prime and represents the size of the finite field $(a, b)$ : the parameters of elliptic curves $y^{2}=x^{3}+a x+b$ over $F(p)$

$G\left(x_{p}, y_{p}\right)$ : generator point, but $G \neq 0$

$n$ : the order of the base point $G$

$h$ : cofactor, an integer, $h=F(p) / n$

Elliptic Curve Diffie-Hellman (ECDH) is an anonymous key agreement protocol that allows two parties; each has an elliptic curve based public, private key pair, to establish a shared secret over an insecure channel. Suppose Alice wants to establish a shared key with Bob, but the channel available for them is not safe. Initially, the domain parameters $(p, a, b, G, n, h)$ must be agreed upon. Also, each party must have a key pair suitable for elliptic curve cryptography, consisting of a private key $d$ (a randomly selected integer in the interval $[1, n-1]$ ) and a public key $Q$ (where $Q=d G$, that is, the result of adding $G$ together $d$ times).

Alice's private key and public key are $\left(d_{A}, Q_{A}\right)$; Bob's key pair is $\left(d_{B}, Q_{B}\right)$. Alice computes $d_{A} Q_{B}$ while Bob computes $d_{B} Q_{A}$. So the shared key between them is $d_{A} Q_{B}=d_{B} Q_{A}$, because

$$
d_{A} Q_{B}=d_{A} d_{B} G=d_{B} d_{A} G=d_{B} Q_{A}
$$

\section{Privacy Enhanced Scheme: PriAuth}

The structure model of our scheme is depicted in Figure 1. A gateway is introduced to help user and sensor authenticate each other. We suppose this gateway is trustworthy.

4.1. Symbols Used in the PriAuth. Before the scheme begins, GWN (gateway node) generates the parameters for ECC encryption $(p, a, b, G, n, h)$. After that, GWN generates its public-key pair $\left(d_{g}, Q_{g}\right)$; besides, GWN generates a secret key $X_{\mathrm{GWN}}$. The symbols are summarized in Table 2 . 


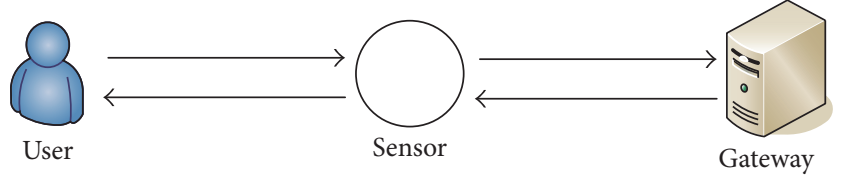

FIGURE 1: The structure of the model.

TABLE 2: Symbols used in the PriAuth.

\begin{tabular}{lc}
\hline Symbols & Meaning \\
\hline GWN & Gateway node \\
$U_{i}$ & The $i$ th user \\
$S_{j}$ & The $j$ th sensor node \\
$\mathrm{ID}_{i}$ & The $i$ th user's identity \\
$\mathrm{SID}_{j}$ & The $j$ th sensor's identity \\
$\|$ & XOR operation \\
$\oplus$ & GWN's secret value, master key \\
$X_{\mathrm{GWN}}$ & Shared key between $S_{j}$ and $\mathrm{GWN}$ \\
$X_{\mathrm{GWN}-S_{j}}$ & The private key and public key of $\mathrm{GWN}$ \\
$\left(d_{g}, Q_{g}\right)$ & The generator of ECC \\
$G$ & Shared key between user $U_{i}$ and $S_{j}$ \\
$\mathrm{SK}_{\mathrm{S}}, \mathrm{SK}^{\prime}$ & Timestamp \\
$T_{1}, T_{2}$ & Hash function \\
$h$ &
\end{tabular}

4.2. Registration Phase of the Sensor. The registration messages of the sensor in registration phase are sent via the public channel. Sensor $S_{j}$ conducts the following steps for registration:

(1) It creates a random number $r_{j}$ and gets the timestamp $T_{1}$.

(2) It covers its password with $r_{j}, M N_{j}=r_{j} \oplus X_{\mathrm{GWN}-S_{j}}$ and generates a hash value $M P_{j}=h\left(X_{\mathrm{GWN}-S_{j}}\left\|r_{j}\right\|\right.$ $\left.\mathrm{SID}_{j} \| T_{1}\right)$.

(3) It sends $\left\{\operatorname{SID}_{j}, M P_{j}, M N_{j}, T_{1}\right\}$ to $\mathrm{GWN}$ via a public channel.

After GWN receives $S_{j}$ 's registration message $\left\{\mathrm{SID}_{j}, M P_{j}, M N_{j}, T_{1}\right\}$. GWN has to check the freshness of the message by $T_{1}$, if the message is not fresh, GWN abandons the message. Then GWN computes $r_{j}^{\prime}=M N_{j} \oplus X_{\mathrm{GWN}-S_{j}}$. GWN checks if $M P_{j}$ equals $h\left(X_{\mathrm{GWN}^{-} S_{j}}\left\|r_{j}^{\prime}\right\| \operatorname{SID}_{j} \| T_{1}\right)$. If they are not equal, GWN abandons the message. GWN continues the sensor registration phase in the following steps. The registration phase is described in Table 3.

(1) GWN computes $x_{j}=h\left(\operatorname{SID}_{j} \| X_{\mathrm{GWN}}\right), e_{j}=x_{j} \oplus$ $h\left(\mathrm{SID}_{j} \| X_{\mathrm{GWN}^{-} S_{j}}\right)$.

(2) GWN gets the timestamp $T_{2}$ and gets the hash value $f_{j}=h\left(x_{j}\left\|X_{\mathrm{GWN} S_{j}}\right\| T_{2}\right)$.

(3) GWN sends $\left\{e_{j}, f_{j}, T_{2}, p, a, b, G, n, h, Q_{g}\right\}$ to sensor $S_{j}$.

After receiving the message, $S_{j}$ first checks the freshness of $T_{2}$, then computes $x_{j}=e_{j} \oplus h\left(\operatorname{SID}_{j} \| X_{\mathrm{GWN}_{-}}\right)$, and checks if $f_{j}=h\left(x_{j}\left\|X_{\mathrm{GWN}-S_{j}}\right\| T_{2}\right)$; if they are equal, $S_{j}$ stores $\left\{x_{j}, p, a, b, G, n, h, Q_{g}\right\}$ in its memory.

4.3. Registration Phase of the User. User $U_{i}$ chooses a random number $r_{i}$ and computes $M P_{i}=h\left(r_{i}\left\|\mathrm{ID}_{i}\right\| \mathrm{PW}_{i}\right) . U_{i}$ then sends $\left\{\mathrm{ID}_{i}, M P_{i}\right\}$ to $\mathrm{GWN}$ via a secure channel.

After receiving the user registration message $\left\{\mathrm{ID}_{i}, M P_{i}\right\}$, GWN computes $d_{i}=h\left(\mathrm{ID}_{i} \| X_{\mathrm{GWN}}\right), f_{i}=d_{i} \oplus M P_{i}$. Finally, GWN sends $\left\{f_{i}, p, a, b, G, n, h, Q_{g}\right\}$ to $U_{i}$.

After receiving $\left\{f_{i}, p, a, b, G, n, h, Q_{g}\right\}, U_{i}$ inserts the previously selected random nonce $r_{i}$ into it, now what in the smart card is $\left\{M P_{i}, f_{i}, r_{i}, p, a, b, G, n, h, Q_{g}\right\}$. The registration phase is described in Table 4.

4.4. Login and Authentication Phase. If user $U_{i}$ wants to access a sensor's data, $U_{i}$ has to login first. This login process is completed by the smart card SC. A user inserts his smart card SC into a card reader and inputs his identity $\mathrm{ID}_{i}^{\prime}$ and password $\mathrm{PW}_{i}^{\prime}$. SC computes a temporary version $M P_{i}^{\prime}=h\left(r_{i}\left\|\mathrm{ID}_{i}^{\prime}\right\|\right.$ $\mathrm{PW}_{i}^{\prime}$ ) using the inserted $\mathrm{PW}_{i}^{\prime}, \mathrm{ID}_{i}^{\prime}$ and the stored value $r_{i}$. Then SC compares $M P_{i}^{\prime}$ with $M P_{i}$ in the smart card. If they are equal, SC acknowledges the legitimacy of $U_{i}$.

After user $U_{i}$ passes through the verification, then SC prepares for the authentication process. SC computes $d_{i}=$ $f_{i} \oplus M P_{i}^{\prime}$ using $M P_{i}^{\prime}$ in login phase. SC chooses a random number $k_{1} \in[1, n-1]$ and gets the timestamp $T_{1}$. SC then computes the following data:

$$
\begin{aligned}
& A=k_{1} \cdot G \\
& K_{u g}=h\left(T_{1} \| k_{1} \cdot Q_{g}\right) \\
& M_{1}=\left(\operatorname{ID}_{i}, \operatorname{SID}_{j}\right) \oplus K_{u g} \\
& M_{2}=h\left(A\left\|M_{1}\right\| d_{i} \| T_{1}\right)
\end{aligned}
$$

Then SC sends Message $1=\left\{A, M_{1}, M_{2}, T_{1}\right\}$ to sensor $S_{j}$ via a public channel.

After receiving $\left\{A, M_{1}, M_{2}, T_{1}\right\}$ from $U_{i}$, sensor $S_{j}$ first checks the freshness of $T_{1}$ and $S_{j}$ abandons the message if $T_{1}$ is not fresh and otherwise goes to the next step. $S_{j}$ chooses a random number $k_{2} \in[1, n-1]$ and gets the timestamp $T_{2} . S_{j}$ then computes the following data:

$$
\begin{aligned}
& B=k_{2} \cdot G \\
& M_{3}=h\left(B\left\|M_{2}\right\| x_{j} \| T_{2}\right)
\end{aligned}
$$

$S_{j}$ sends Message $2=\left\{A, M_{1}, M_{2}, T_{1}, B, M_{3}, T_{2}\right\}$ to GWN via a public channel.

After receiving the message $\left\{A, M_{1}, M_{2}, T_{1}, B, M_{3}, T_{2}\right\}$, GWN first checks the freshness of $T_{1}$ and $T_{2}$, if $T_{1}$ or $T_{2}$ is not fresh, GWN abandons the message; otherwise GWN completes the following steps:

(1) GWN computes $K_{u g}^{\prime}=h\left(T_{1} \| d_{g} \cdot A\right)$.

(2) GWN gets $\operatorname{ID}_{i}^{\prime}$ and $\operatorname{SID}_{j}^{\prime}$ by $\left(\operatorname{ID}_{i}^{\prime}, \operatorname{SID}_{j}^{\prime}\right)=M_{1} \oplus K_{u g}^{\prime}$.

(3) GWN computes $d_{i}^{\prime}$ by $d_{i}^{\prime}=h\left(\mathrm{ID}_{i}^{\prime} \| X_{\mathrm{GWN}}\right)$.

(4) GWN computes $x_{j}^{\prime}$ by $x_{j}^{\prime}=h\left(\operatorname{SID}_{j}^{\prime} \| X_{\mathrm{GWN}}\right)$. 
TABLE 3: Registration phase of the sensor.

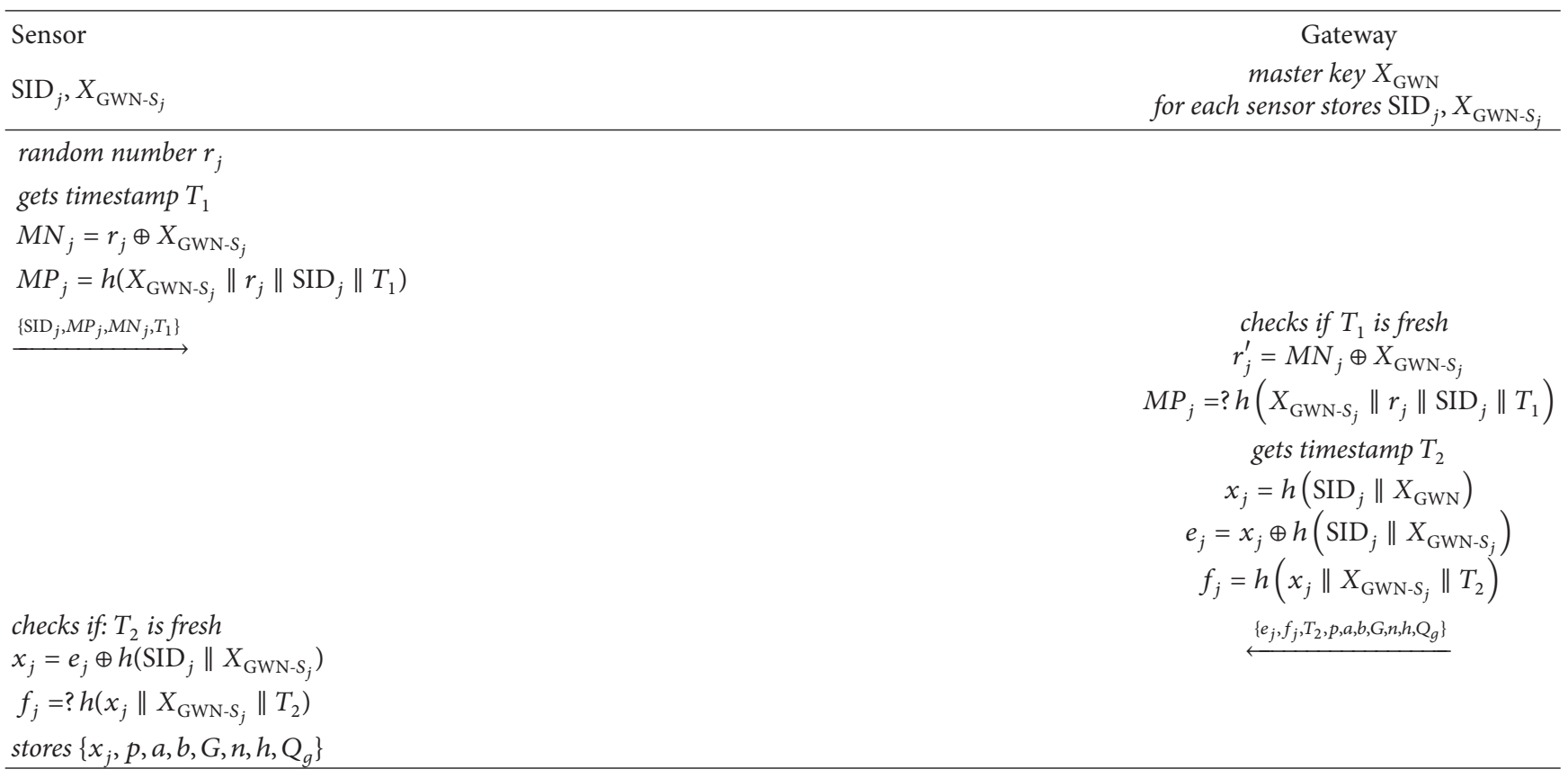

TABLE 4: Registration phase of the user.

\begin{tabular}{|c|c|}
\hline User & Gateway \\
\hline $\mathrm{ID}_{i}, \mathrm{PW}_{i}$ & master key $X_{\mathrm{GWN}}$ \\
\hline \multicolumn{2}{|l|}{ random number $r_{i}$} \\
\hline \multicolumn{2}{|l|}{$M P_{i}=h\left(r_{i}\left\|\mathrm{ID}_{i}\right\| \mathrm{PW}_{i}\right)$} \\
\hline$\stackrel{\left\{\mathrm{ID}_{i}, M P_{i}\right\}}{\longrightarrow}$ & $\begin{array}{c}d_{i}=h\left(\mathrm{ID}_{i} \| X_{\mathrm{GWN}}\right) \\
f_{i}=d_{i} \oplus M P_{i}\end{array}$ \\
\hline $\begin{array}{l}\text { inserts into the smart card } \\
\left\{M P_{i}, f_{i}, r_{i}, p, a, b, G, n, h, Q_{g}\right\}\end{array}$ & $\left\{f_{i}, p, a, b, G, n, h, Q_{g}\right\}$ \\
\hline
\end{tabular}

(5) GWN uses $d_{i}^{\prime}, A, M_{1}$ and $T_{1}$ to check if $M_{2}=h(A \|$ $\left.M_{1}\left\|d_{i}^{\prime}\right\| T_{1}\right)$. If they are equal, the procedure goes to next step; otherwise it terminates here.

(6) GWN uses $x_{j}^{\prime}, B, M_{2}$ and $T_{2}$ to check if $M_{3}=h(B \|$ $M_{2}\left\|x_{j}^{\prime}\right\| T_{2}$ ). If they are equal, the procedure goes to next step; otherwise it terminates here.

(7) GWN calculates the following messages:

$$
\begin{aligned}
& M_{4}=h\left(A\left\|x_{j}\right\| M_{3}\|B\| T_{2}\right) \\
& M_{5}=h\left(B\left\|d_{i}\right\| M_{2}\|A\| T_{1}\right)
\end{aligned}
$$

(8) GWN sends Message $3=\left\{M_{4}, M_{5}\right\}$ to sensor $S_{j}$.

After receiving the message $\left\{M_{4}, M_{5}\right\}$, sensor $S_{j}$ does the following calculations:

(1) $S_{j}$ uses $A$ getting from user to checks if $M_{4}=h(A \|$ $x_{j}\left\|M_{3}\right\| B \| T_{2}$ ). If they are equal, the procedure goes to next step; otherwise it terminates here.

(2) $S_{j}$ calculates the shared key SK between $U_{i}$ and $S_{j}$ : $\mathrm{SK}=h\left(k_{2} \cdot A\right)=h\left(k_{1} \cdot k_{2} \cdot G\right)$.

(3) $S_{j}$ sends Message $4=\left\{B, M_{5}\right\}$ to user $U_{i}$
After $U_{i}$ receives the message $\left\{B, M_{5}\right\}, U_{i}$ goes to the following steps. The whole process is in Table 5 .

(1) $U_{i}$ uses $B$ getting from $S_{j}$ to check if $M_{5}=h\left(B\left\|d_{i}\right\|\right.$ $\left.M_{2}\|A\| T_{1}\right)$; if they are equal, the procedure goes to next step; otherwise it terminates here.

(2) $U_{i}$ calculates the shared key $\mathrm{SK}^{\prime}$ between $U_{i}$ and $S_{j}$ : $\mathrm{SK}^{\prime}=h\left(k_{1} \cdot B\right)=h\left(k_{1} \cdot k_{2} \cdot G\right)$.

4.5. Password Change Phase. If a user wants to change his password, he has to be authenticated by the smart card first. We state the password change process in Table 6 , which is a summary of the steps:

(1) A user $U_{i}$ inserts his smart card SC into a card reader and inputs their identity and password: $\mathrm{ID}_{i}, \mathrm{PW}_{i}$.

(2) SC computes $h\left(r_{i}\left\|\mathrm{ID}_{i}\right\| \mathrm{PW}_{i}\right)$ using password $\mathrm{ID}_{i}$, $\mathrm{PW}_{i}$, and the stored $r_{i}$.

(3) SC compares $h\left(r_{i}\left\|\mathrm{ID}_{i}\right\| \mathrm{PW}_{i}\right)$ with the stored version of $M P_{i}$ in the smart card; if they are equal, SC acknowledges the legitimacy of user $U_{i}$. 
TABLE 5: Login and authentication phase.

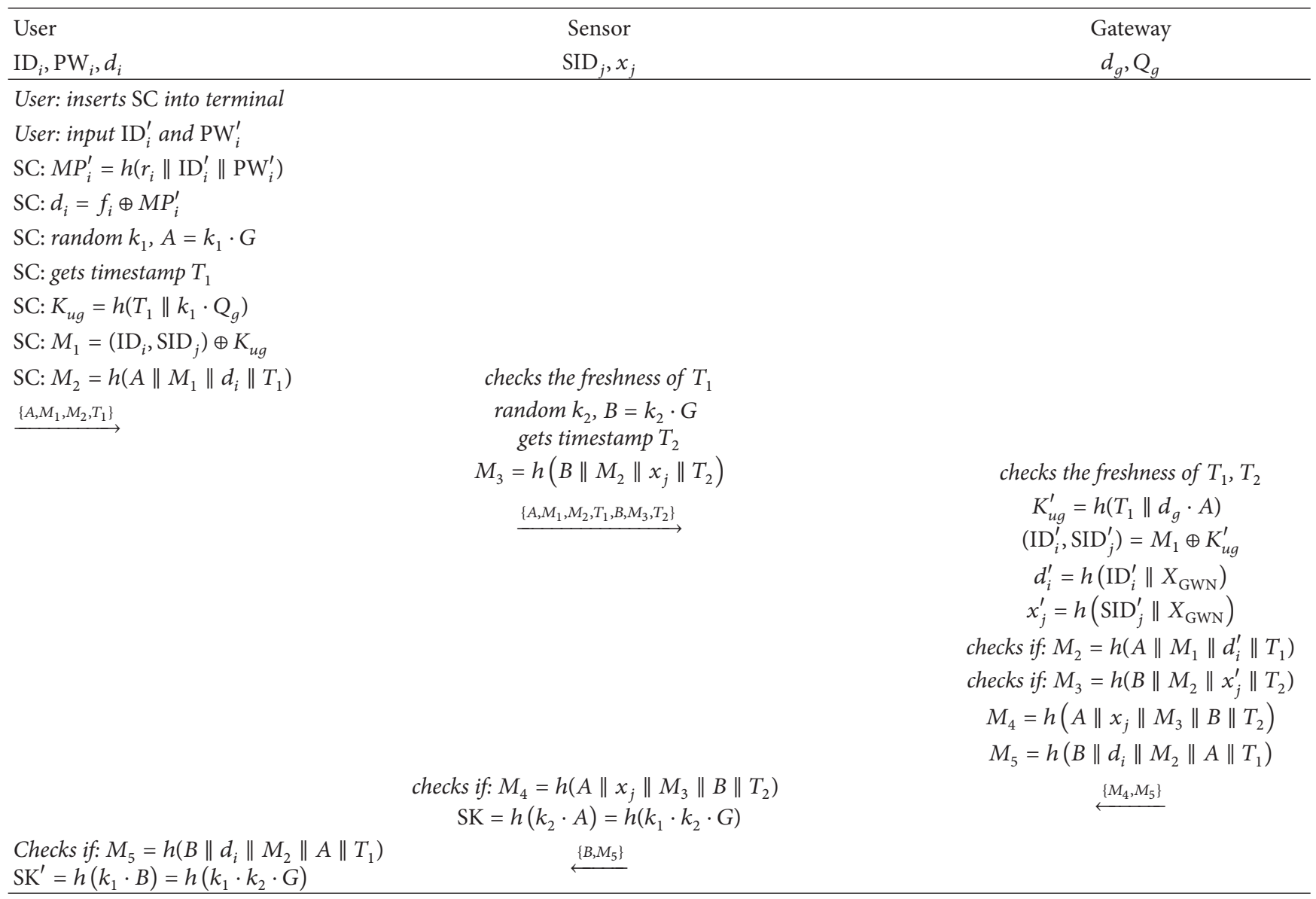

TABLE 6: Password change phase of the user.

\begin{tabular}{l}
\hline User \\
\hline User: inserts $\mathrm{SC}$ into terminal \\
User: inserts $\mathrm{ID}_{i}$ and $\mathrm{PW}_{i}$ \\
SC: check if $M P_{i}=$ ? $h\left(r_{i}\left\|\mathrm{ID}_{i}\right\| \mathrm{PW}_{i}\right)$ \\
SC: $d_{i}=f_{i} \oplus M P_{i}$ \\
User: inputs a new password $\mathrm{PW}_{i}^{\prime}$ \\
SC: $M P_{i}^{\prime}=h\left(r_{i}\left\|\mathrm{ID}_{i}\right\| \mathrm{PW}_{i}^{\prime}\right)$ \\
SC: $f_{i}^{\prime}=d_{i} \oplus M P_{i}^{\prime}$ \\
SC: changes $f_{i}$ with $f_{i}^{\prime}$
\end{tabular}

(4) SC computes $d_{i}=f_{i} \oplus M P_{i}$ using the stored values $f_{i}$ and the user password $M P_{i}$.

(5) User $U_{i}$ inputs the new password $\mathrm{PW}_{i}^{\prime}$.

(6) SC uses this new $\mathrm{PW}_{i}^{\prime}$ to update the stored version of $f_{i}$ with $f_{i}^{\prime}=d_{i} \oplus M P_{i}^{\prime}$.

\section{Security Analysis Using BAN Logic}

5.1. Some Basic Knowledge of BAN Logic. A security analysis of PriAuth using Burrows-Abadi-Needham logic (BAN logic) [41] is conducted in this part. With the help of BAN logic,
TABLE 7: Symbols of BAN logic.

\begin{tabular}{lc}
\hline Symbol & Meaning \\
\hline$P \mid \equiv X$ & $P$ believes $X$ \\
$P \triangleleft X$ & $P$ sees/receives $X$ \\
$P \mid \sim X$ & $P$ once said $X$ (or $P$ sent $X)$ \\
$P \mid \Rightarrow X$ & $P$ controls $X$ \\
$\#(X)$ & $X$ is fresh \\
$P \stackrel{k}{\leftrightarrow} Q$ & $P$ and $Q$ communicate using shared key $K$ \\
$\stackrel{k}{\rightarrow} Q$ & $K$ is the public key of $Q$ \\
$\{X\}_{k}$ & Message $X$ is encrypted by $K$ \\
$\{X\}_{k^{-1}}$ & Message $X$ is encrypted by private key $K$ \\
\hline
\end{tabular}

we can determine whether the exchanged information is trustworthy and secure against eavesdropping. First, some symbols and primary postulates used in BAN logic are described in Tables 7 and 8.

5.2. The Premise and Proof Goals of PriAuth. $U_{i}, S_{j}$, and GWN are used as the user, sensor, and the gateway. Suppose GWN is trustworthy, if GWN believes that $U_{i}$ has said message $X$ and GWN believes that $X$ is fresh, GWN would send $X$ to $S_{j}$. If $S_{j}$ believes $X$ is fresh and $S_{j}$ believes GWN once said $X$, then $S_{j}$ believes $U_{i}$ said $X$. This could be translated into BAN logic 
TABLE 8: Some primary BAN logic postulates.

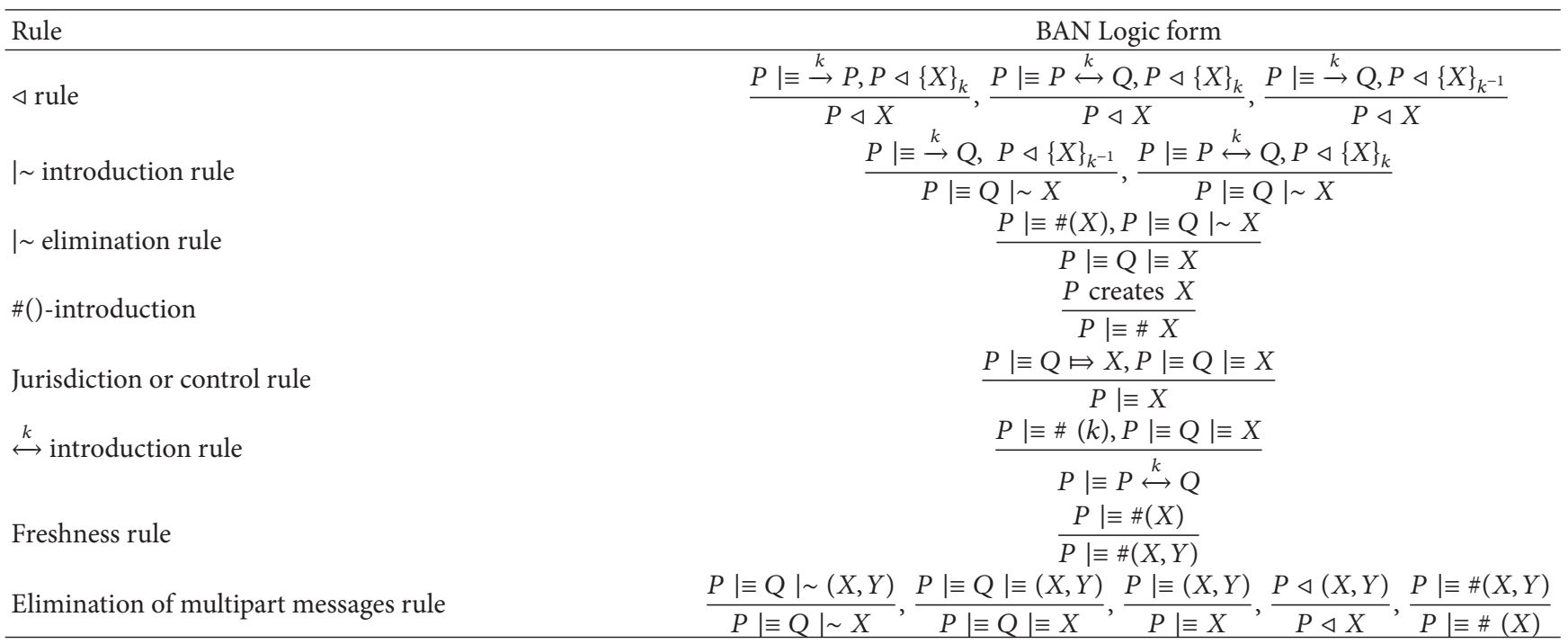

like (postulate A). According to the " $\mid$ elimination rule," (postulate A) could be simplified as (postulate B). It is the same as the message that sensor $S_{j}$ sends to GWN. If GWN believes $S_{j}$ once said another message $X$ (the same notion is used for simplification), and GWN believes $X$ is fresh, GWN would send $X$ to $U_{i}$. If $U_{i}$ believes $X$ is fresh and $U_{i}$ believes GWN once said $X$, then $U_{i}$ believes $S_{j}$ said $X$. In the same way, we can get (postulate $C$ ).

$$
\begin{gathered}
\frac{\mathrm{GWN}|\equiv \#(X), \mathrm{GWN}| \equiv U_{i}\left|\sim X, S_{j}\right| \equiv \#(X), S_{j}|\equiv \mathrm{GWN}| \sim X}{S_{j}\left|\equiv U_{i}\right| \sim X} \\
\frac{\mathrm{GWN}\left|\equiv U_{i}\right| \equiv X, S_{j}|\equiv \mathrm{GWN}| \equiv X}{S_{j}\left|\equiv U_{i}\right| \sim X} \\
\frac{\mathrm{GWN}\left|\equiv S_{j}\right| \equiv X, U_{i}|\equiv \mathrm{GWN}| \equiv X}{U_{i}\left|\equiv S_{j}\right| \sim X}
\end{gathered}
$$

(postulate A)

(postulate B)

(postulate C)

The proof goals of PriAuth in BAN logic form are in the way described below. These goals could ensure $U_{i}$ and $S_{j}$ to agree on a shared key SK.

$$
\begin{aligned}
& \text { (1) } U_{i} \mid \equiv U_{i} \stackrel{\mathrm{SK}}{\longleftrightarrow} S_{j} \\
& \text { (2) } S_{j} \mid \equiv U_{i} \stackrel{\mathrm{SK}}{\longleftrightarrow} S_{j} .
\end{aligned}
$$

5.3. Preparation for Proof. Before the proof begins, messages have to be transformed into an idealized form, the messages of PriAuth in idealized form in BAN logic are given in Table 9 $\left(K_{u g}=h\left(T_{1} \| k_{1} \cdot Q_{g}\right)\right)$. At the same time, some assumptions have to be made, so (postulate B) and (postulate $C$ ) are included as assumptions A11 and A12. The assumptions are listed in Table 10.

5.4. The Proof of PriAuth. The whole proof of the proposal is in Appendix A. It has been divided into 3 parts related to Message 2, Message 3, and Message 4 separately. The two goals of the scheme are proved at the Message 3 and Message 4. The proof results show that PriAuth is secured under BAN logic.

\section{AVISPA Verification}

AVISPA (Automated Validation of Internet Security Protocols and Applications) is "a push-button tool for the automated validation of Internet security-sensitive protocols and applications" [42]. Recently, many papers have used this method as a way to authenticate their protocols, like [24-26]. HLPSL (High Level Protocols Specification Language) is a role-based language that is used to describe security protocols and specifying their intended security properties, as well as a set of tools to formally validate them. We write the protocol in HLPSL and test the protocol. The code is in Appendix B. The goal of PriAuth is to create a key that is shared by a user and a sensor. The validation result of the protocol is in Table 11. Considering all these testing activities, it could be concluded that our protocol is safe. PriAuth can protect the privacy of the user identity, sensor identity, and the key between the user and sensor. 
TABLE 9: The idealization form of the message.

\begin{tabular}{lcc}
\hline Message & Flow & Idealized form \\
\hline 1 & $U_{i} \longrightarrow S_{j}$ & $\left\{A,\left\{\operatorname{ID}_{i}, \operatorname{SID}_{j}\right\}_{K_{u g}},\left\{A,\left\{\operatorname{ID}_{i}, \operatorname{SID}_{j}\right\}_{K_{u g}}, T_{1}\right\}_{d_{i}}, T_{1}\right\}$ \\
2 & $S_{j} \longrightarrow \mathrm{GWN}$ & $\left\{A,\left\{\operatorname{ID}_{i}, \operatorname{SID}_{j}\right\}_{K_{u g}},\left\{A,\left\{\operatorname{ID}_{i}, \operatorname{SID}_{j}\right\}_{K_{u g}}, T_{1}\right\}_{d_{i}}, T_{1}, B,\left\{B, M_{2}, T_{2}\right\}_{x_{j}}, T_{2}\right\}$ \\
3 & $\mathrm{GWN} \rightarrow S_{j}$ & $\left\{\left\{A, M_{3}, B, T_{2}\right\}_{x_{j}},\left\{B, M_{2}, A, T_{1}\right\}_{d_{i}}\right\}$ \\
4 & $S_{j} \longrightarrow U_{i}$ & $\left\{B,\left\{B, M_{2}, A, T_{1}\right\}_{d_{i}}\right\}$ \\
\hline
\end{tabular}

TABLE 10: Some assumptions.

\begin{tabular}{|c|c|}
\hline Number & Assumptions \\
\hline A1 & $\mathrm{GWN} \mid \equiv \#(A)$ \\
\hline A2 & $\mathrm{GWN} \mid \equiv \#(B)$ \\
\hline A3 & $S_{j} \mid \equiv \#(A)$ \\
\hline A4 & $U_{i} \mid \equiv \#(B)$ \\
\hline A5 & $U_{i} \mid \equiv \mathrm{GWN} \stackrel{d_{i}}{\longleftrightarrow} U_{i}$ \\
\hline A6 & $\mathrm{GWN} \mid \equiv \mathrm{GWN} \stackrel{d_{i}}{\longleftrightarrow} U_{i}$ \\
\hline A7 & $U_{i} \mid \equiv \mathrm{GWN} \stackrel{K_{u g}}{\longleftrightarrow} U_{i}$ \\
\hline A8 & $\mathrm{GWN} \mid \equiv \mathrm{GWN} \stackrel{K_{u g}}{\longleftrightarrow} U_{i}$ \\
\hline A9 & $S_{j} \mid \equiv \mathrm{GWN} \stackrel{x_{j}}{\longleftrightarrow} S_{j}$ \\
\hline $\mathrm{A} 10$ & $\mathrm{GWN} \mid \equiv \mathrm{GWN} \stackrel{x_{j}}{\longleftrightarrow} S_{j}$ \\
\hline \multirow{2}{*}{ A11 } & $\mathrm{GWN}\left|\equiv U_{i}\right| \equiv X, S_{j}|\equiv \mathrm{GWN}| \equiv X$ \\
\hline & $\begin{array}{c}S_{j}\left|\equiv U_{i}\right| \sim X \\
\mathrm{GWN}\left|\equiv S_{j}\right| \equiv X, U_{i}|\equiv \mathrm{GWN}| \equiv X\end{array}$ \\
\hline A12 & $\begin{array}{c}U_{i}\left|\equiv S_{j}\right| \sim X \\
\end{array}$ \\
\hline $\mathrm{A} 13$ & $S_{j} \mid \equiv U_{i} \Leftrightarrow A$ \\
\hline A14 & $U_{i} \mid \equiv S_{j} \Leftrightarrow B$ \\
\hline
\end{tabular}

\section{Security and Privacy Analysis}

In this section, we conduct a security comparison of the schemes that has been depicted as Table 12. For the scheme in [3], we only consider the second situation.

7.1. Traceability Protection. Traceability means the adversary can track a user or a sensor according to their identities or masked identities like in the scheme [5, 10, 29-32]. Once some fixed information about the identities is used in a scheme, then this scheme could probably be tracked by an adversary. One possible solution is to update their masked identity every time like in the schemes shown in $[4,7]$. But these kinds of solutions are vulnerable to loss of synchronization attack.

7.2. Synchronization Loss Attack. In order to protect the identity of the user, the gateway will generate a new identity for them when it is requested [4]. But if an adversary prevents this new identity from being received by the user, the user could not update his old identity while the gateway has updated its stored version of the user's identity. When the user logs in for the next time, this legitimate user will not be treated as a legal one anymore. A similar problem exists in the scheme [7].
7.3. Malicious Sensor Attack. Like in scheme [13], the gateway only checks the legitimacy of a sensor. If the sensor is a legitimate one, the gateway will reply some key information to the sensor, but the gateway does not check if the sensor is the one that the user wants to talk to. So a legitimate but malicious sensor could launch an attack.

When a user sends a request message $\left\{M_{1}, M_{2}, M_{3}, T_{1}\right\}$ to a sensor, an inner side legitimate sensor can intercept this message to generate its own $\left\{M_{4}^{\prime}, M_{5}^{\prime}, \mathrm{ESID}_{j}^{\prime}, T_{2}^{\prime}\right\}$ and send this message to the gateway, as the gateway only checks the legitimacy of the sensor. Therefore, this inner side sensor will definitely be treated as a legal sensor. The gateway will send $\left\{M_{6}^{\prime}, M_{7}^{\prime}, M_{8}^{\prime}, M_{9}^{\prime}, T_{3}^{\prime}\right\}$ to the sensor. Afterwards, the sensor will be able to send $\left\{M_{6}^{\prime}, M_{8}^{\prime}, M_{10}^{\prime}, T_{3}^{\prime}, T_{4}^{\prime}\right\}$ to the user, and it will be treated as a legal sensor by the user, but the user will not check if this is the sensor he wants to talk to. In this way, the sensor could send false data to the user.

7.4. Inside User Attack. In scheme [6], all the users share a key $v^{*}$, so there is a potential risk. The message a gateway sends to the user is $D_{i}=E_{k}\left(\mathrm{DID}_{i}\left\|\mathrm{SID}_{n}\right\| \mathrm{SK}\left\|R_{1}\right\| T_{4}\right)$, where $k=$ $h\left(\mathrm{DID}_{i}\left\|v^{*}\right\| T_{4}\right)$, in which $\mathrm{DID}_{i}$ and $T_{4}$ are public message, and $v^{*}$ is shared by all the legitimate users. This means any legitimate user could decrypt $D_{i}$ to get the shared key SK.

7.5. User Impersonation Attack. In scheme [1], when a user asks to access a sensor's data, he could send his request $M_{1}=$ $\left\{\mathrm{ID}_{u}, \mathrm{ID}_{S_{n}}, X, T_{u}, \alpha, \omega\right\}$ to the sensor.

$$
\begin{aligned}
X^{\prime} & =r_{u} \times P, \\
X & =r_{u} \times K_{u}, \\
\omega & =h\left(\mathrm{ID}_{u}\left\|h\left(\mathrm{ID}_{S_{n}} \| h(X \oplus Y)\right)\right\| T_{u}\right), \\
\alpha & =h\left(\mathrm{ID}_{u}\left\|\mathrm{ID}_{S_{n}}\right\| X\left\|X^{\prime}\right\| T_{u} \| \omega\right) .
\end{aligned}
$$

$\mathrm{ID}_{u}, K_{u}, P$, and $\mathrm{ID}_{S_{n}}$ are sent publicly; $r_{u}$ is a random number generated by the user, whereas $T_{u}$ is a timestamp. Only $h(X \oplus Y)$ is regarded as secret information between the user and the gateway. $h(X \oplus Y)$ is shared by all the users; other legitimate users, say a legitimate user with $\mathrm{ID}_{u}^{\prime}$, could easily generate a request the same as $M_{1}$, and then $\mathrm{ID}_{u}^{\prime}$ will be treated as $\mathrm{ID}_{u}$ by the gateway.

\section{Comparison}

8.1. Computational Performance. The normal way to compute the execution time of the protocol is to calculate protocol's 
TABLE 11: Simulation results.

\begin{tabular}{|c|c|}
\hline CL-AtSe back-end & OFMC \\
\hline SUMMARY & $\%$ OFMC \\
\hline \multirow[t]{2}{*}{ SAFE } & $\%$ Version of $2006 / 02 / 13$ \\
\hline & SUMMARY \\
\hline DETAILS & SAFE \\
\hline BOUNDED_NUMBER_OF_SESSIONS & DETAILS \\
\hline \multirow[t]{2}{*}{ TYPED_MODEL } & BOUNDED_NUMBER_OF_SESSIONS \\
\hline & PROTOCOL \\
\hline \multicolumn{2}{|l|}{ PROTOCOL } \\
\hline & /home/iotdev/avispa/avispa-1.1/testsuite/results/usg.if \\
\hline /home/iotdev/avispa/avispa-1.1/testsuite/results/usg.if & GOAL \\
\hline GOAL & as_specified \\
\hline \multirow[t]{2}{*}{ As Specified } & BACKEND \\
\hline & OFMC \\
\hline BACKEND & COMMENTS \\
\hline \multirow[t]{2}{*}{ CL-AtSe } & STATISTICS \\
\hline & parseTime: $0.00 \mathrm{~s}$ \\
\hline STATISTICS & searchTime: $0.05 \mathrm{~s}$ \\
\hline Analysed: 14 states & visitedNodes: 24 nodes \\
\hline Reachable: 4 states & depth: 4 plies \\
\hline \multicolumn{2}{|l|}{ Translation: 0.00 seconds } \\
\hline Computation: 0.00 seconds & \\
\hline
\end{tabular}

TABLE 12: Security feature comparison.

\begin{tabular}{lccccc}
\hline Security feature & {$[1]$} & {$[3$, Scheme 2] } & {$[7]$} & {$[9]$} & PriAuth \\
\hline User anonymity & $\times$ & $\times$ & $\sqrt{ }$ & $\sqrt{ }$ & $\times$ \\
Sensor anonymity & $\times$ & $\times$ & $\times$ & $\sqrt{ }$ & $\sqrt{ }$ \\
Shared key privacy & $\sqrt{ }$ & $\sqrt{ }$ & $\sqrt{ }$ & $\times \sqrt{ }$ \\
Traceability of user & $\times$ & $\times$ & $\times$ & $\sqrt{ }$ \\
Traceability of sensor & $\times$ & $\times$ & $\sqrt{ }$ \\
Loss of synchronization & $\sqrt{ }$ & $\sqrt{ }$ & $\sqrt{ }$ \\
Malicious sensor attack & $\sqrt{ }$ & $\sqrt{ }$ & $\sqrt{ }$ \\
User impersonation attack & $\times$ & $\sqrt{ }$ & $\sqrt{ }$ \\
Sensor impersonation attack & $\sqrt{ }$ & $\sqrt{ }$ & $\sqrt{ }$ \\
Replay attack & $\sqrt{ }$ & $\sqrt{ }$ & $\sqrt{ }$ \\
Inside user attack & $\sqrt{ }$ & $\sqrt{ }$ & $\sqrt{ }$ \\
\hline
\end{tabular}

computational costs of different operations, and the operations' execution time is measured by simulation [3-14]. The execution time of XOR operation is very small compared to an elliptic curve point multiplication or hash operation; we neglect it when computing the time approximately [3]. We use the famous MIRACL++ Library [43] (example code can be found at [44]). The experiment is conducted in Visual $\mathrm{C}++2017$ on a 64-bit Windows 7 operating system, $3.5 \mathrm{GHz}$ processor, $8 \mathrm{~GB}$ memory. The hash function is the SHA-1; the symmetric encryption/decryption function is AES with a 128-bit long key of the MR_PCFB1 form (using one string to encrypt another string, the same hash function is called to get the hashed form of the key string). The elliptic curve encryption scheme is ECC-160. The results are shown in
Table 13. $T_{\text {mac }}$ is the time for HMAC with SHA-1 operation, according to [9] $T_{\mathrm{mac}} \approx T_{H}$. The final result is in Table 14 .

8.2. Communication Performance. The sum of each variable length in bytes which a sensor node and a gateway node need while performing authentication process is calculated for comparison of the communication cost. The identity or password is 8-byte long [13]. The sizes of the general hash function's output and timestamp are 20 bytes and 4 bytes, respectively [45]. The random point of ECC-160 is 20 bytes. The result is shown in Table 15. The byte length of the AES encryption result is treated as byte length of the original data for approximation. 
TABLE 13: Computation time of different operations.

\begin{tabular}{lcc}
\hline Operations & Time & Experiment times \\
\hline$T_{H}:$ one way hash function & $0.0394 \mathrm{~ms}$ & 1000000 \\
$T_{E / D}:$ symmetric encryption/decryption & $0.5728 \mathrm{~ms}$ & 100000 \\
$T_{\text {MUL }}:$ scalar multiplication in ECC-160 & $3.66 \mathrm{~ms}$ & 2733 \\
\hline
\end{tabular}

\section{Validation}

LifeWear project intends to improve the quality of human life by using wearable equipment and applications for everyday use [46]. The main objective of LifeWear is the development of modern physiological monitoring to inspect human health parameters, like blood pressure, pulse, or the electrocardiogram of a patient in different environments. With realtime data of these health parameters, medical staffs can take actions instantly, which can greatly improve the quality of a treatment.

Since medical parameters are sent from patients to medical staffs, data security and patient's privacy are a must. In order to ensure the data confidentiality, all the data must be encrypted before they are sent. The proposed scheme helps the patients and medical staff building a shared key. This key will be used to encrypt the health parameters of the patient. In order to protect the privacy of the patient, all the identities are encrypted before they are sent as well. Since wearable sensors have only limited computability, we introduce a gateway to provide the patients and medical staff the shared key to be used in the system.

LifeWear project also makes use of a middleware solution able to hide heterogeneity and interoperability problem. This middleware is composed of four abstraction layers related to the functionalities covered in each of them, namely, hardware abstraction layer, low and high services, cross-layer services, and service composition platform.

The hardware abstraction layer includes the IoT hardware platform, the operating system, and the networking stack. It offers an easy way to port the solution to other hardware platforms. The low and high service layers define the software components needed to abstract the underlying network heterogeneity, thus providing an integrated, distributed environment to simplify programming tasks by means of a set of generic services, along with an access point to the management functions of the sensor network services. The upper layer is the service composition platform, designed to build applications using services offered by the lower layers. The cross-layer services are offered to both high and low level services in order to provide inner service composition. The proposal presented in this paper (PriAuth) has been deployed as a service inside this layer. The security service can be used by the upper layer (service composition) to compose newly secured services, based on the services presented in the lower layers.

The architecture has been deployed over a commercial IoT node solution called SunSPOT platform, manufactured by Oracle. Main characteristics of SunSPOT hardware platform are as follows: (a) Processor: ARM 920T CPU (400 MHz, 32 bits)

(b) Memory: 1 Mb RAM, 8 Mb Flash memory

(c) Network: Chipcon 2420 radio with integrated antenna (IEEE 802.15.4 at $2.4 \mathrm{GHz}$ )

(d) Data: USB interface, mini-USB connector

(e) Power supply: $3.6 \mathrm{~V}$ rechargeable $750 \mathrm{mAh}$ Li-Ion battery

\section{Conclusions}

Privacy will be a big concern as more and more IoT equipment is applied into the medical scenarios. In this paper, we propose an authentication and key agreement scheme tailored for Wireless Sensor Networks. We focus on the privacy problems during the authentication process. Our scheme not only ensures the security of the data but also protects the identity privacy of the users and sensors. The shared key between the user and sensor is built by means of the Elliptic Curve Diffie-Hellman method, which could ensure forward privacy. The proposed scheme has been verified with BAN logic and AVISPA, which are the two most commonly used tools to validate the security of the communication scheme. Simulation results show that our scheme is feasible and secure. Furthermore, experiment results show that our scheme is comparable with the related works in terms of computation cost and more efficient in communication cost.

As part of our work in the LifeWear project, we focus on privacy problems during the authentication and key establishment processes. In future, we will pay more attention to authentication scheme without the help of the gateway.

\section{Appendix}

\section{A. The Proof of PriAuth Using BAN Logic}

The proof starts at Message 2. From Message 2 onwards, we can prove that GWN believes $U_{i}$ once said $A$ and GWN believes $S_{j}$ once said $B$.

(1) According to Message 2, we get

$$
\begin{aligned}
& \mathrm{GWN} \triangleleft\left\{A,\left\{\mathrm{ID}_{i}, \mathrm{SID}_{j}\right\}_{K_{u g}},\right. \\
& \left.\quad\left\{A,\left\{\mathrm{ID}_{i}, \mathrm{SID}_{j}\right\}_{K_{u g}}, T_{1}\right\}_{d_{i}}, T_{1}, B,\left\{B, M_{2}, T_{2}\right\}_{x_{j}}, T_{2}\right\} .
\end{aligned}
$$




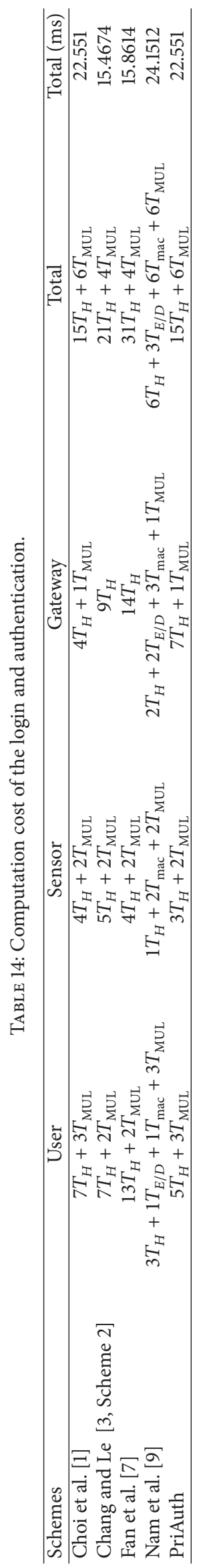


TABLE 15: Communication comparison.

\begin{tabular}{lcccccc}
\hline Schemes & M1 & M2 & M3 & M4 & Total bytes & Compared $^{*}$ \\
\hline Choi et al. [1] & 80 & 124 & 44 & 68 & 316 & +64 \\
Chang and Le [3, Scheme 2] & 64 & 84 & 64 & 44 & 100 & 356 \\
Fan et al. [7] & 128 & 68 & 60 & 56 & 252 & +4 \\
Nam et al. [9] & 52 & 104 & 40 & 40 & 252 & 0 \\
PriAuth & 64 & 108 & 40 &
\end{tabular}

Compared* means compared with our scheme; M1, M2, M3, and M4 mean Messages 1, 2, 3, and 4.

(2) According to (A.1) and "',-elimination rule"

$$
\begin{aligned}
& \mathrm{GWN} \triangleleft\left\{A,\left\{\mathrm{ID}_{i}, \mathrm{SID}_{j}\right\}_{K_{u g}}, \mathrm{SID}_{j}, T_{1}\right\}_{d_{i}}, \\
& \mathrm{GWN} \triangleleft\left\{B, M_{2}, T_{2}\right\}_{x_{j}} .
\end{aligned}
$$

(3) According to (A.2), A6, and "| introduction rule"

$$
\mathrm{GWN}\left|\equiv U_{i}\right| \sim\left\{A,\left\{\mathrm{ID}_{i}, \mathrm{SID}_{j}\right\}_{K_{u g}}, \mathrm{SID}_{j}, T_{1}\right\} .
$$

(4) According to (A.3), A10, and "I introduction rule"

$$
\mathrm{GWN}\left|\equiv S_{j}\right| \sim\left\{B, M_{2}, T_{2}\right\} \text {. }
$$

(5) According to (A.4) and "“, --elimination rule”

$$
\mathrm{GWN}\left|\equiv U_{i}\right| \sim A \text {. }
$$

(6) According to (A.5) and “', --elimination rule”

$$
\mathrm{GWN}\left|\equiv S_{j}\right| \sim B \text {. }
$$

(7) According to A1, (A.6), and "| elimination rule"

$$
\mathrm{GWN}\left|\equiv U_{i}\right| \equiv A \text {. }
$$

(8) According to A2, (A.7), and "I elimination rule"

$$
\mathrm{GWN}\left|\equiv S_{j}\right| \equiv B \text {. }
$$

The following content is the analysis of Message 3. From it, we can prove that $S_{j}$ believes GWN believes $A$. Based on assumption A11, we can get that $S_{j}$ believes $U_{i}$ believes $A$; this process is shown at (A.10) (A.17). Equations (A.18) (A.20) prove the first goal of the scheme.

(9) Based on Message 3,

$$
S_{j} \triangleleft\left\{\left\{A, M_{3}, B, T_{2}\right\}_{x_{j}},\left\{B, M_{2}, A, T_{1}\right\}_{d_{i}}\right\} .
$$

(10) According to (A.10) and “',-elimination rule”

$$
S_{j} \triangleleft\left\{\left\{A, M_{3}, B, T_{2}\right\}_{x_{j}}\right\} .
$$

(11) According to (A.11), A9, and "| introduction rule"

$$
S_{j}|\equiv \mathrm{GWN}| \sim\left\{A, M_{3}, B, T_{2}\right\} .
$$

(12) According to (A.12) and "',-elimination rule"

$$
S_{j}|\equiv \mathrm{GWN}| \sim A \text {. }
$$

(13) According to A3, (A.13), and "| elimination rule"

$$
S_{j}|\equiv \mathrm{GWN}| \equiv A \text {. }
$$

(14) According to A11, (A.8), (A.14), we get

$$
S_{j}\left|\equiv U_{i}\right| \sim A \text {. }
$$

(15) According to A3, (A.15), and " $\mid$ elimination rule"

$$
S_{j}\left|\equiv U_{i}\right| \equiv A \text {. }
$$

(16) According to A13, (A.16), and "jurisdiction or control rule"

$$
S_{j} \mid \equiv A
$$

(17) As $k_{2}$ is randomly created by $S_{j}$, according to “\#()introduction"

$$
S_{j} \mid \equiv \#\left(k_{2}\right)
$$

(18) According to (A.18), A3, A5, and “\#()-promotion rule"

$$
S_{j} \mid \equiv \#(\mathrm{SK}) \quad \mathrm{SK}=h\left(k_{2} \cdot A\right)
$$

(19) According to (A.19), (A.17), and “ $\stackrel{k}{\leftrightarrow}$ introduction rule"

$$
S_{j} \mid \equiv S_{j} \stackrel{\mathrm{SK}}{\longleftrightarrow} U_{i}
$$

The following is the analysis of Message 4, where it is proven that $U_{i}$ believes GWN and believes $B$, based on assumption A12, so we can infer that $U_{i}$ believes $S_{j}$ believes $B$; this procedure is shown at (A.21) (A.28). Equations (A.29) (A.31) prove the first goal of the scheme. Until now, the two goals of the scheme have been proved at (A.20) and (A.31), so it can be claimed that this protocol is feasible and safe.

(20) Based on Message 4,

$$
U_{i} \triangleleft\left\{B,\left\{B, M_{2}, A, T_{1}\right\}_{d_{i}}\right\} .
$$




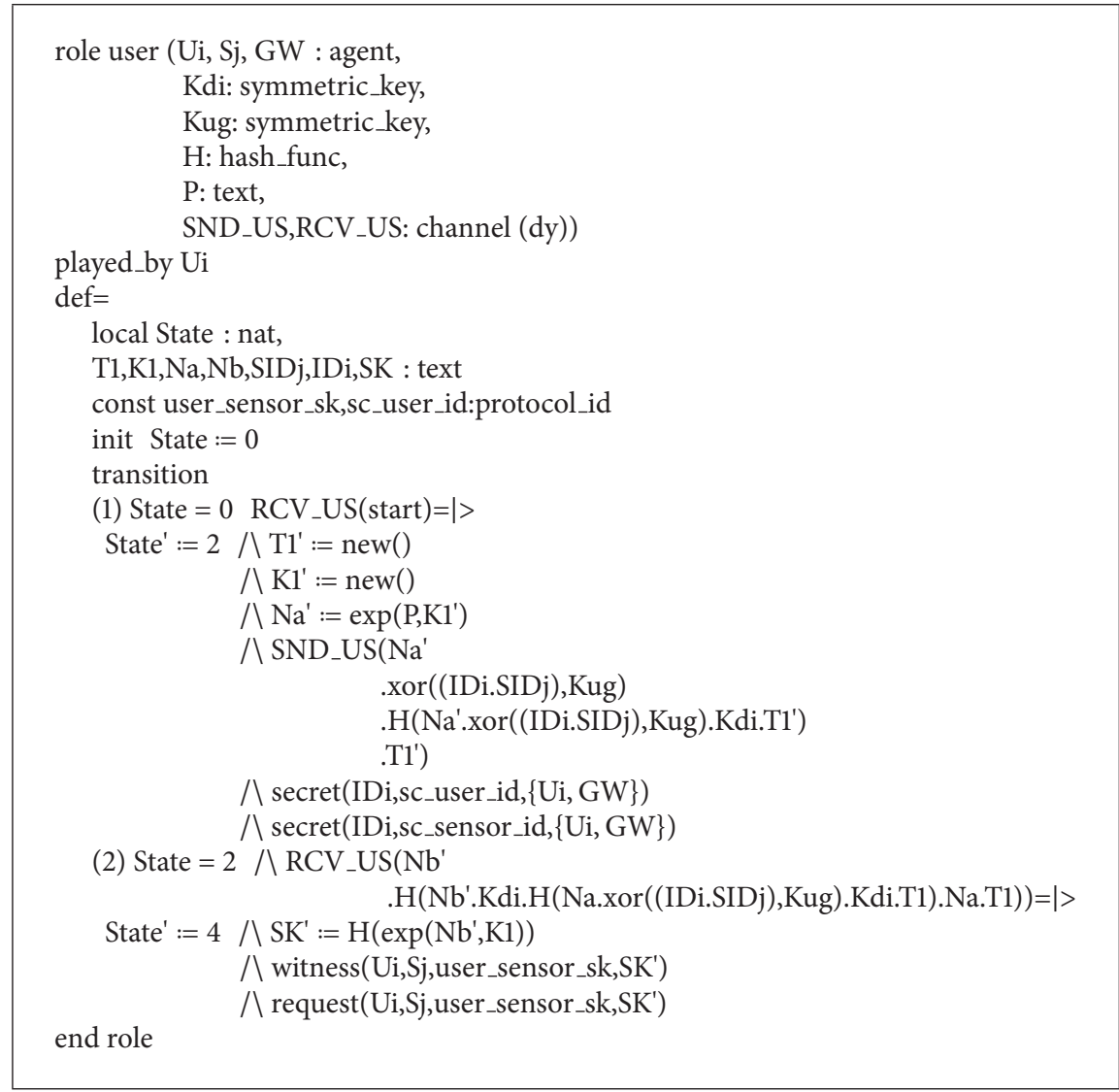

Box 1

(21) According to (A.21) and “',-elimination rule”

$$
U_{i} \triangleleft\left\{\left\{B, M_{2}, A, T_{1}\right\}_{d_{i}}\right\} .
$$

(22) According to (A.22), A7, and "| introduction rule"

$$
U_{i}|\equiv \mathrm{GWN}| \sim\left\{B, M_{2}, A, T_{1}\right\} .
$$

(23) According to (A.23) and “",-elimination rule”

$$
U_{i}\left|\equiv S_{j}\right| \sim B
$$

(24) According to A4, (A.23), and "| elimination rule"

$$
U_{i}|\equiv \mathrm{GWN}| \equiv B
$$

(25) According to A12, (A.9), and (A.25), we get

$$
U_{i}\left|\equiv S_{j}\right| \sim B
$$

(26) According to A4, (A.26), and "| elimination rule"

$$
U_{i}\left|\equiv S_{j}\right| \equiv B
$$

(27) According to A14, (A.27), and "jurisdiction or control rule"

$$
U_{i} \mid \equiv B
$$

(28) As $k_{2}$ is randomly created by $U_{i}$, according to "\#()introduction"

$$
U_{i} \mid \equiv \#\left(k_{1}\right)
$$

(29) According to (A.29), A4, A6, and “\#()-promotion rule"

$$
U_{i} \mid \equiv \#(\mathrm{SK}) \quad \mathrm{SK}=h\left(k_{1} \cdot B\right) .
$$

(30) According to (A.30), (A.27), and “ $\stackrel{k}{\longleftrightarrow ~ i n t r o d u c t i o n ~}$ rule"

$$
U_{i} \mid \equiv S_{j} \stackrel{\mathrm{SK}}{\longleftrightarrow} U_{i}
$$

\section{B. The HLPSL Code for PriAuth}

The ECC public-key pair of the gateway is $\left(d_{g}, Q_{g}\right)$. At the beginning of this protocol usage, every user generates a random number $k_{1} \in[1, n-1]$ and calculates $A=k_{1} \cdot G$, so we could treat $\left(k_{1}, A\right)$, as the ECC key pair of this user, and we send $A$ to the gateway. Now the two parties could calculate a shared key $k_{1} \cdot Q_{g}=d_{g} \cdot A$. Thus, at the beginning of the scheme, we declare $K_{u g}=h\left(T_{1} \| k_{1} \cdot Q_{g}\right)$ to be a symmetric key between the two.

For the role of the user, see Box 1. For the role of the sensor, see Box 2. For the role of the gateway, see Box 3 . 
role sensor $(\mathrm{Ui}, \mathrm{Sj}, \mathrm{GW}$ : agent,

Kxj: symmetric_key,

H: hash_func,

P: text,

SND_US,RCV_US,SND_SG,RCV_SG: channel(dy))

played_by $S j$

def $=$

local State : nat,

$\mathrm{T} 1, \mathrm{~T} 2, \mathrm{~K} 2, \mathrm{Na}, \mathrm{Nb}, \mathrm{SK}$ : text,

$\mathrm{Y}, \mathrm{X}, \mathrm{Z}$ : message

const user_sensor_sk:protocol_id

init State $:=1$

transition

(1) State $=1 /$ RCV_US(Na'.Y'.Z'.T1') $=\mid>$

State' $^{\prime}:=3 \wedge \mathrm{T}^{\prime}:=\operatorname{new}()$

$\bigwedge \mathrm{K} 2{ }^{\prime}:=\operatorname{new}()$

$\bigwedge \mathrm{Nb}^{\prime}:=\exp \left(\mathrm{P}, \mathrm{K} 2^{\prime}\right)$

$\bigwedge$ SND_SG $\left(\mathrm{Na}^{\prime}\right.$

$\mathrm{Y}^{\prime}$

$\mathrm{Z}^{\prime}$

.T1'

. $\mathrm{Nb}$

$\mathrm{H}\left(\mathrm{Nb} \cdot \mathrm{Z}^{\prime} . \mathrm{Kxj} . \mathrm{T2} 2^{\prime}\right)$

.T2')

(2) State $=2 /$ RCV_SG( H(Na.Kxj.H(Nb.Z.Kxj.T2).T2)

.$\left.X^{\prime}\right)=\mid>$

State' $:=4 \quad \bigwedge \mathrm{SK}^{\prime}:=\mathrm{H}(\exp (\mathrm{Na}, \mathrm{K} 2))$

$\bigwedge$ witness(Sj,Ui,user_sensor_sk,SK')

$\bigwedge$ request(Sj,Ui,user_sensor_sk,SK')

$\bigwedge$ SND_US(Nb

end role

. $\left.\mathrm{X}^{\prime}\right)$

Box 2

role gateway $(\mathrm{Ui}, \mathrm{Sj}, \mathrm{GW}$ : agent,

Kdi, Kxj: symmetric_key,

Kug : symmetric_key,

$\mathrm{H}$ : hash_func,

SND_SG, RCV_SG: channel(dy))

played_by GW

def $=$

local State : nat,

T1,T2,Na,Nb,IDi,SIDj : text

const sk_User_gwn,sk_sensor_gwn,sc_sensor_id,sc_user_id:protocol_id init State $:=5$

transition

(1) State $=5 /$ RCV_SG $\left(\mathrm{Na}^{\prime}\right.$

.xor((IDi'.SIDj'),Kug)

.H(Na'.xor((IDi'.SIDj'),Kug).Kdi.T1')

.T1'

. $\mathrm{Nb}^{\prime}$

.H(Nb'.H(Na'.xor((IDi'.SIDj'),Kug).Kdi.T1').Kxj.T2')

.T2') $=\mid>$

State' $:=7$ 八 SND_SG(

H(Na'.Kxj.H(Nb'.H(Na'.xor((IDi'.SIDj'),Kug).Kdi.T1').Kxj.T2').T2')

.H(Nb'.Kdi.H(Na'.xor((IDi'.SIDj'),Kug).Kdi.T1').Na'.T1)

$\bigwedge \operatorname{secret}(I D i '$, sc_user_id, $\{$ Ui, GW $\})$

end role

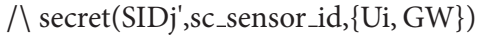




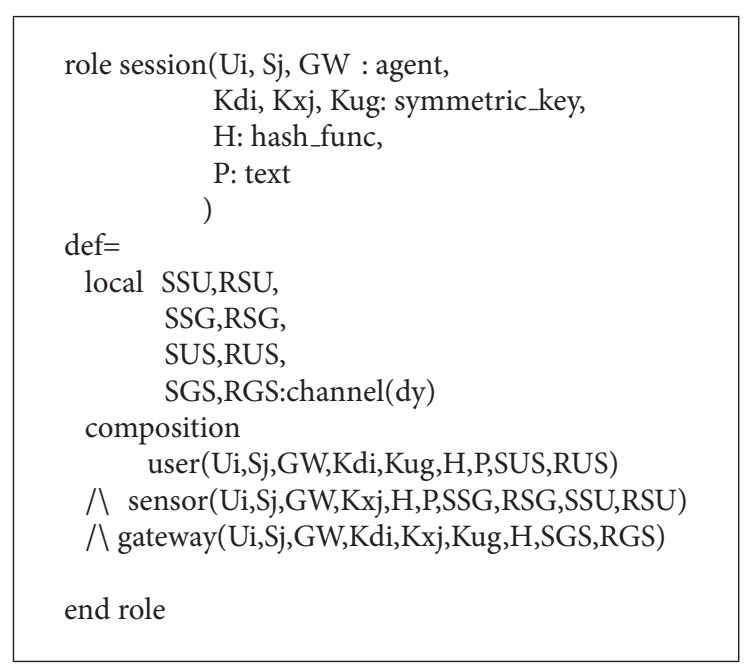

Box 4

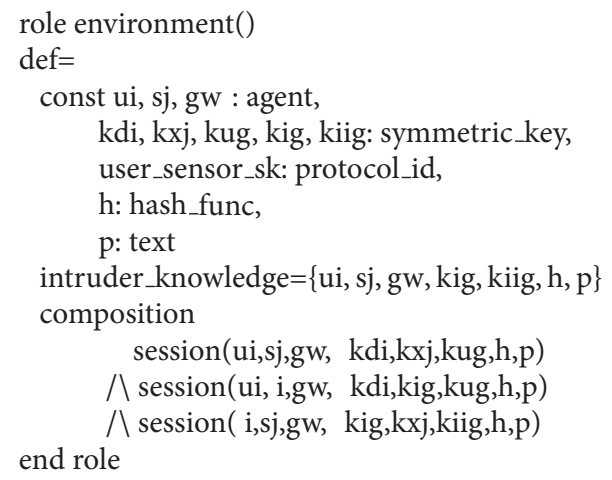

Box 5

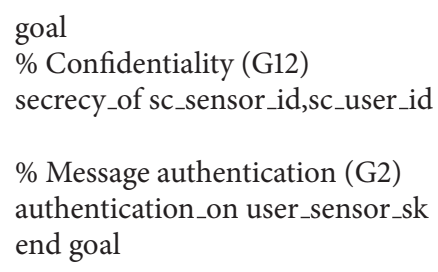

Box 6

For the role of the session, see Box 4. For the role of the environment, see Box 5.

The role of the goal is divided into two parts. The first part is the "secrecy_of sc_sensor_id,sc_user_id"; this means we want to keep the identity of the user and sensor confidential between them and the gateway. The second part "authentication_on user_sensor_sk" means the authentication of the shared key between a user and a sensor (see Box 6).

\section{Conflicts of Interest}

The authors declare no conflicts of interest.

\section{Authors' Contributions}

All the authors have contributed equally to this work.

\section{Acknowledgments}

The work presented in this paper has been supported by the LifeWear Project (funded by the Spanish Ministry of Industry, Energy and Tourism with Reference TSI-0104002010-100). The work has also been supported by the Chinese Scholarship Council (CSC) with File no. 201507040027.

\section{References}

[1] Y. Choi, D. Lee, and J. Kim, "Security enhanced user authentication protocol for wireless sensor networks using elliptic curves cryptography," Sensors, vol. 14, no. 6, pp. 10081-10106, 2014.

[2] W. B. Shi and P. Gong, "A new user authentication protocol for wireless sensor networks using elliptic curves cryptography," International Journal of Distributed Sensor Networks, vol. 2013, Article ID 730831, 7 pages, 2013.

[3] C.-C. Chang and H.-D. Le, "A Provably secure, efficient, and flexible authentication scheme for ad hoc wireless sensor networks," IEEE Transactions on Wireless Communications, vol. 15, no. 1, pp. 357-366, 2016.

[4] F. Wu et al., "A Novel and provably secure authentication and key agreement scheme with user anonymity for global mobility networks," Security and Communication Networks, vol. 9, no. 16, pp. 3527-3542, 2016.

[5] A. K. Das et al., "Provably secure user authentication and key agreement scheme for wireless sensor networks," Security and Communication Networks, vol. 9, no. 16, pp. 3670-3687, 2016.

[6] J. Jung, J. Kim, Y. Choi, and D. Won, "An anonymous user authentication and key agreement scheme based on a symmetric cryptosystem in wireless sensor networks," Sensors, vol. 16, no. 8, article 1299, 2016.

[7] W. Fan et al., "A privacy-preserving and provable user authentication scheme for wireless sensor networks based on internet of things security," Journal of Ambient Intelligence and Humanized Computing, pp. 1-16, 2016.

[8] R. Amin and G. Biswas, "A secure light weight scheme for user authentication and key agreement in multi-gateway based wireless sensor networks," Ad Hoc Networks, vol. 36, part 1, pp. 58-80, 2016.

[9] J. Nam, M. Kim, J. Paik, Y. Lee, and D. Won, "A provablysecure ECC-based authentication scheme for wireless sensor networks," Sensors, vol. 14, no. 11, pp. 21023-21044, 2014.

[10] Y. Lu, L. Li, H. Peng, and Y. Yang, "An energy efficient mutual authentication and key agreement scheme preserving anonymity for wireless sensor networks," Sensors, vol. 16, no. 6, p. 837, 2016.

[11] D. Zhao, H. Peng, L. Li, and Y. Yang, "A secure and effective anonymous authentication scheme for roaming service in global mobility networks," Wireless Personal Communications, vol. 78, no. 1, pp. 247-269, 2014.

[12] J. L. Hou et al., "Novel Authentication Schemes for IoT Based Healthcare Systems, Novel Authentication Schemes for IoT 
Based Healthcare Systems," International Journal of Distributed Sensor Networks, Article ID e183659, 2015.

[13] M. S. Farash, M. Turkanović, S. Kumari, and M. Hölbl, "An efficient user authentication and key agreement scheme for heterogeneous wireless sensor network tailored for the Internet of Things environment," Ad Hoc Networks, vol. 36, pp. 152-176, 2016.

[14] M. Turkanović, B. Brumen, and M. Hölbl, "A novel user authentication and key agreement scheme for heterogeneous ad hoc wireless sensor networks, based on the Internet of Things notion," Ad Hoc Networks, vol. 20, pp. 96-112, 2014.

[15] S. Chatterjee and A. K. Das, "An effective ECC-based user access control scheme with attribute-based encryption for wireless sensor networks," Security and Communication Networks, vol. 8, no. 9, pp. 1752-1771, 2015.

[16] D. Mishra, A. K. Das, and S. Mukhopadhyay, "A secure and efficient ECC-based user anonymity-preserving session initiation authentication protocol using smart card," Peer-to-Peer Networking and Applications, vol. 9, no. 1, pp. 171-192, 2016.

[17] Q. Jiang, N. Kumar, J. Ma, J. Shen, D. He, and N. Chilamkurti, "A privacy-aware two-factor authentication protocol based on elliptic curve cryptography for wireless sensor networks," International Journal of Network Management, vol. 27, no. 3, Article ID e1937, 2017.

[18] Q. Jiang, J. Ma, F. Wei, Y. Tian, J. Shen, and Y. Yang, "An untraceable temporal-credential-based two-factor authentication scheme using ECC for wireless sensor networks," Journal of Network and Computer Applications, vol. 76, pp. 37-48, 2016.

[19] J. Nam, K.-K. R. Choo, S. Han, M. Kim, J. Paik, and D. Won, "Efficient and anonymous two-factor user authentication in wireless sensor networks: achieving user anonymity with lightweight sensor computation," PLOS ONE, vol. 10, no. 4, Article ID e0116709, 2015.

[20] J. Moon, H. Yang, Y. Lee, and D. Won, "Improvement of user authentication scheme preserving uniqueness and anonymity for connected health care," in Proceedings of the 11th International Conference on Ubiquitous Information Management and Communication (IMCOM '17), Japan, January 2017.

[21] A. G. Reddy, A. K. Das, E.-J. Yoon, and K.-Y. Yoo, "A secure anonymous authentication protocol for mobile services on elliptic curve cryptography," IEEE Access, vol. 4, pp. 4394-4407, 2016.

[22] N. Saxena, B. J. Choi, and R. Lu, "Authentication and authorization scheme for various user roles and devices in smart grid," IEEE Transactions on Information Forensics and Security, vol. 11, no. 5, pp. 907-921, 2016.

[23] H. Ning, H. Liu, and L. T. Yang, "Aggregated-proof based hierarchical authentication scheme for the internet of things," IEEE Transactions on Parallel and Distributed Systems, vol. 26, no. 3, pp. 657-667, 2015.

[24] V. Odelu, A. K. Das, and A. Goswami, "A secure biometricsbased multi-server authentication protocol using smart cards," IEEE Transactions on Information Forensics and Security, vol. 10, no. 9, pp. 1953-1966, 2015.

[25] A. Rossi, S. Pierre, and S. Krishnan, "Secure route optimization for MIPv6 using enhanced CGA and DNSSEC," IEEE Systems Journal, vol. 7, no. 3, pp. 351-362, 2013.

[26] V. Odelu, A. K. Das, and A. Goswami, "SEAP: secure and efficient authentication protocol for NFC applications using pseudonyms," IEEE Transactions on Consumer Electronics, vol. 62, no. 1, pp. 30-38, 2016.
[27] D. Wang and P. Wang, "Understanding security failures of two-factor authentication schemes for real-time applications in hierarchical wireless sensor networks," Ad Hoc Networks, vol. 20, pp. 1-15, 2014.

[28] D. Wang and P. Wang, "On the anonymity of two-factor authentication schemes for wireless sensor networks: attacks, principle and solutions," Computer Networks, vol. 73, pp. 41-57, 2014.

[29] P. Kumar, A. Gurtov, M. Ylianttila, S.-G. Lee, and H. J. Lee, "A strong authentication scheme with user privacy for wireless sensor networks," ETRI Journal, vol. 35, no. 5, pp. 889-899, 2013.

[30] M. K. Khan and S. Kumari, "An improved user authentication protocol for healthcare services via wireless medical sensor networks," International Journal of Distributed Sensor Networks, vol. 2014, Article ID 347169, 10 pages, 2014.

[31] J. Moon, Y. Choi, J. Jung, and D. Won, "An improvement of robust biometrics-based authentication and key agreement scheme for multi-server environments using smart cards," PLoS ONE, vol. 10, no. 12, Article ID e0145263, 2015.

[32] M. Alizadeh et al., "Cryptanalysis and improvement of a secure password authentication mechanism for seamless handover," PLOS One, vol. 10, no. 11, Article ID e0142716, 2015.

[33] A. K. Das, A. K. Sutrala, V. Odelu, and A. Goswami, "A secure smartcard-based anonymous user authentication scheme for healthcare applications using wireless medical sensor networks," Wireless Pers Commun, pp. 1-35, 2016.

[34] Q. Jiang, S. Zeadally, J. Ma, and D. He, "Lightweight threefactor authentication and key agreement protocol for internetintegrated wireless sensor networks," IEEE Access, vol. 5, pp. 3376-3392, 2017.

[35] K. H. Rosen, Elementary number theory and its applications, Addison-Wesley Publishing Company, Advanced Book Program, Reading, MA, Second edition, 1988.

[36] D. He, N. Kumar, M. K. Khan, and J.-H. Lee, "Anonymous twofactor authentication for consumer roaming service in global mobility networks," IEEE Transactions on Consumer Electronics, vol. 59, no. 4, pp. 811-817, 2013.

[37] A. K. Das, P. Sharma, S. Chatterjee, and J. K. Sing, "A dynamic password-based user authentication scheme for hierarchical wireless sensor networks," Journal of Network and Computer Applications, vol. 35, no. 5, pp. 1646-1656, 2012.

[38] A. Das, "A secure and effective biometric-based user authentication scheme for wireless sensor networks using smart card and fuzzy extractor," International Journal of Communication Systems, vol. 30, no. 1, Article ID e2933, 2017.

[39] Y. Chung, S. Choi, Y. S. Lee, N. Park, and D. Won, "An enhanced lightweight anonymous authentication scheme for a scalable localization roaming service in wireless sensor networks," Sensors, vol. 16, no. 10, article 1653, 2016.

[40] Commercial National Security Algorithm Suite and Quantum Computing FAQ U.S. National Security Agency, January 2016.

[41] M. Burrows, M. Abad, and M. Needham, "A logic of authentication," Proceedings of the Royal Society A Mathematical, Physical and Engineering Sciences, vol. 426, no. 1871, pp. 233-271, 1989.

[42] A. Armando, D. Basin, Y. Boichut et al., "The AVISPA tool for the automated validation of internet security protocols and applications," in Computer Aided Verification: International Conference on Computer Aided Verification, vol. 3576, pp. 281285, Springer, Berlin, Germany, 2005.

[43] 2017, https://www.miracl.com/.

[44] 2017, https://github.com/miracl/MIRACL. 
[45] D. He, S. Zeadally, B. Xu, and X. Huang, "An efficient identitybased conditional privacy-preserving authentication scheme for vehicular ad hoc networks," IEEE Transactions on Information Forensics and Security, vol. 10, no. 12, pp. 2681-2691, 2015.

[46] J. Rodríguez-Molina, J.-F. Martínez, P. Castillejo, and L. López, "Combining wireless sensor networks and semantic middleware for an internet of things-based sportsman/woman monitoring application," Sensors, vol. 13, no. 2, pp. 1787-1835, 2013. 


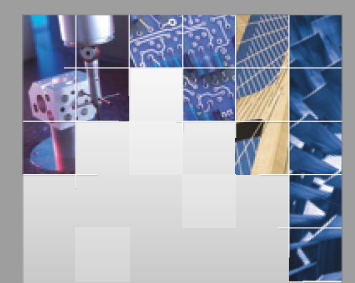

\section{Enfincering}
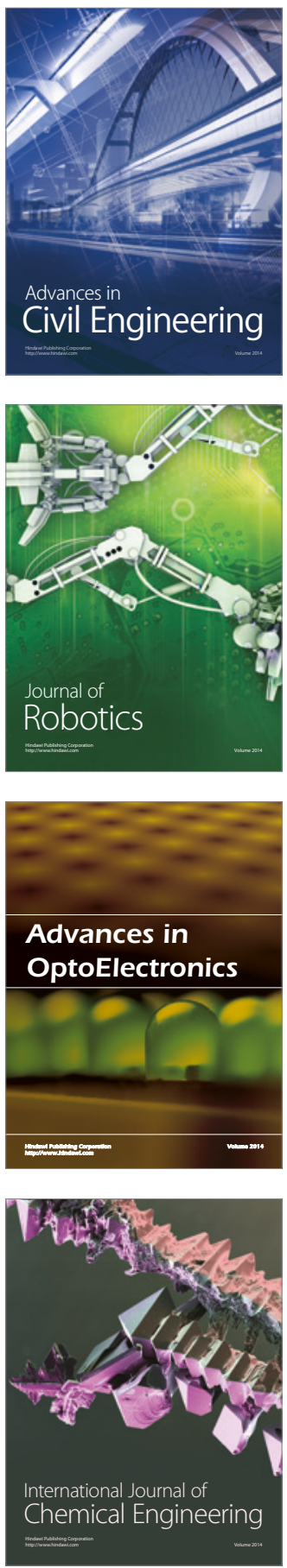

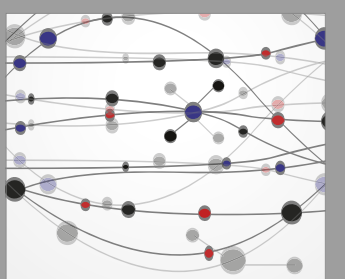

The Scientific World Journal

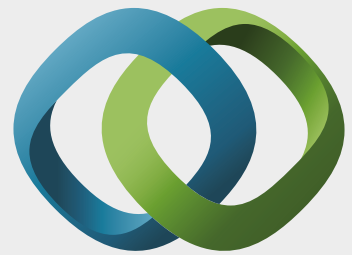

\section{Hindawi}

Submit your manuscripts at

https://www.hindawi.com
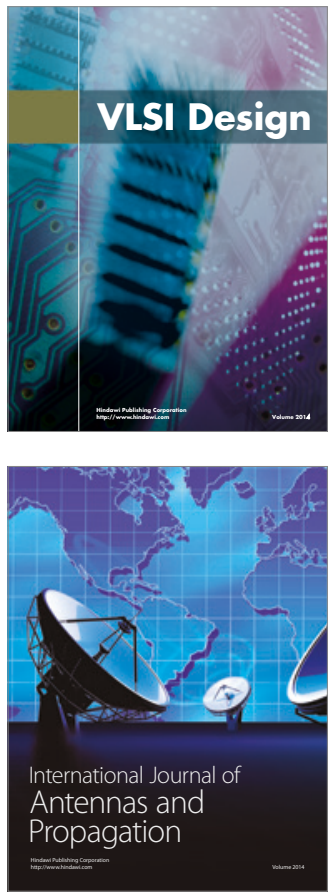

\section{Rotating}

Machinery
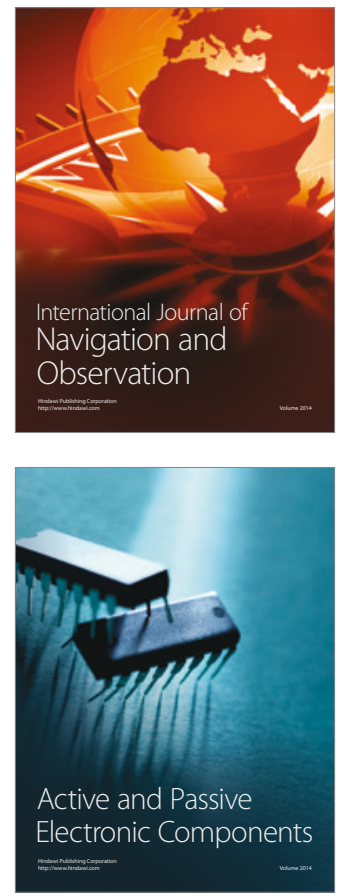
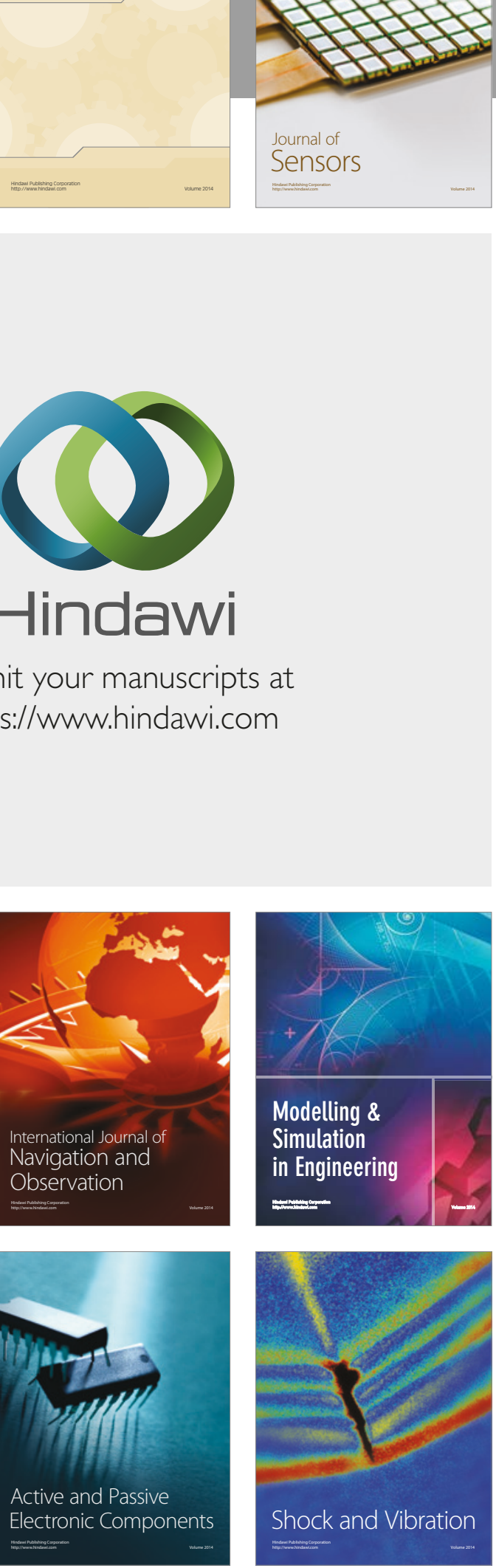
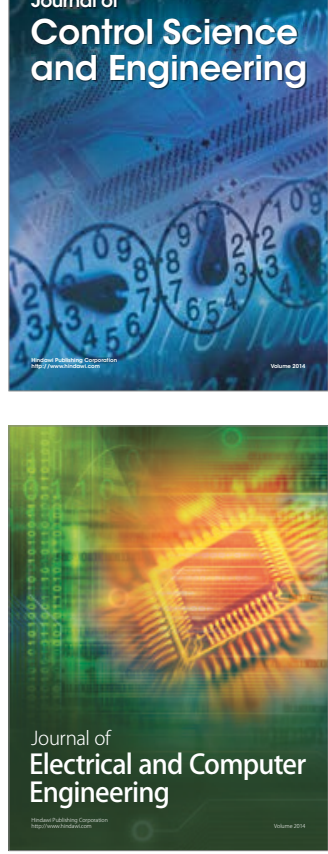

Distributed

Journal of

Control Science

and Engineering
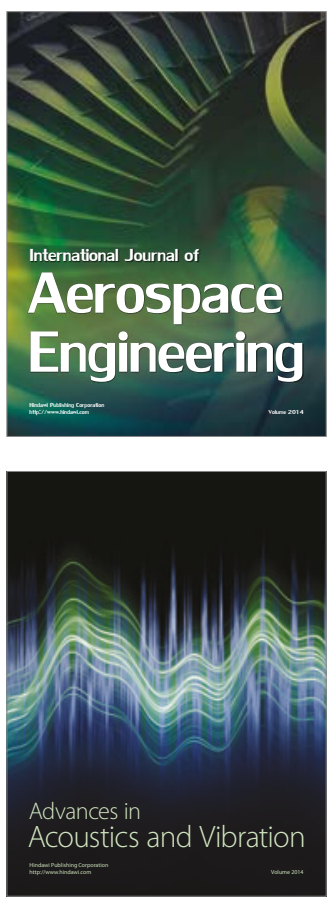

Sensor Networks 